adolescents' school functioning and the pain management interventions in school settings for adolescents with chronic pain.

Methods A systematic review was conducted on school functioning in adolescents aged 13-18 years with chronic pain and pain management in the school setting. A comprehensive search of seven databases; Medline, CINAHL, PsycINFO, ERIC, ScienLO, Cochrane Library, and EMBASE databases, was accomplished covering the period from January $1^{\text {st }}, 1976$ to December $31^{\text {st }}, 2018$.

Results The studies on the topic of school functioning reported that chronic pain has a significant influence on school attendance. Adolescents with chronic pain appear to miss more days than healthy peers. In addition, chronic pain among adolescents is associated with a sense of isolation. This population see themselves as different than their school classmates and peers. However, other studies indicate that chronic pain has no effect on adolescents' academic competence. In order to overcome the struggles of adolescents with chronic pain face in the school settings, studies suggested that the medical team and family members should work in tandem with the teachers to enable an appropriate response to children's chronic pain.

\section{P139 PARTIAL TRISOMY 3P - A CASE REPORT}

Niofa Canty ${ }^{*}$, Aoife Branagan, Evanne O'Halloran, Michael B O’Neill, Hilary Stokes. Department of Paediatrics, Mayo University Hospital, Castlebar, Co Mayo, Ireland

\subsection{6/archdischild-2019-epa.494}

Introduction Partial trisomy $3 \mathrm{p}$ results from an unbalanced translocation or de novo duplication. Clinical features include of structural brain abnormalities, dysmorphic facies, congenital heart defects, learning disability and hypoplastic genitalia. We report the clinical manifestations of a case of partial trisomy $3 \mathrm{p}$.

Case report The female proband, now 3 years and 9 month female was born to a 33 year old Caucasian mother with 2 neurodevelopmentally normal male children. Intrauterine growth restriction and microcephaly identified antenatally prompted investigation. Fetal MRI at 21 weeks gestation demonstrated dilation of the occipital horn of the right lateral ventricle. Amniocentesis confirmed partial trisomy of chromosome 3

The infant was born at term with no resuscitation or respiratory support required. Echocardiogram revealed a secundum atrial septal defect, muscular ventricular septal defect and a restrictive patent ductus arteriosus. Neonatal MRI demonstrated several structural abnormalities:

1. A parieto-occipital encephalocele measuring $2 \times 3.7 \mathrm{~cm}$ containing brain tissue and cerebrospinal fluid

2. Dilation of the occipital horn

3. Bilateral atresia of lateral ventricles

4. Areas of cortical malformation

5. An abnormal corpus callosum

Abdominal and hip ultrasound examination were normal.

The encephalocele was successfully repaired in the neonatal period. Post operative recovery was complicated by development of hydrocephalus requiring a ventriculo-peritoneal shunt. At 10 and 18 months the patient had seizures related to intercurrent illness and fever. She has never had an afebrile seizure.

At present this child is 3 years 9 months with global developmentally delay in all domains. She can walk unaided but has difficulty running. She can use stairs with support but has not progressed to cycling a tricycle. She is visually impaired secondary to a cortical impairment, requiring corrective lenses. She has bilateral sensorineural hearing loss with a cochlear implant. E has remained medically well to this point and has avoided hospital admissions. She requires intensive multidisciplinary involvement by community allied health professionals including physiotherapy, speech and language therapy and occupational therapy. Her parents hope she will be able to access mainstream education with support.

Given the rarity of her condition with few reported cases in the medical literature, her long term developmental outcome remains unclear.

\section{REFERENCES}

1. Partial $3 p$ trisomy and different rearrangements involving chromosome 3 in the proposita's family, De Pinto Neto et al, American Journal of Medical Genetics, 1980

\section{P140 8P 23 DELETION: A CASE REPORT ON AN INFANT WITH HYPOTONIA}

Muhammad Zia*, Raef Abouelhussein, Conn Sreenan, Niazy Alasaaf, Rizwan Khan. University Maternity Hospital Limerick, Limerick, Ireland

10.1136/archdischild-2019-epa.495

Background An infant with hypotonia and dysmorphic features.

Case report A pre term baby boy born to Irish traveller parents at 32 weeks gestation. Mom was Gravida 5 Para 4 with history of oligohydramnios during pregnancy.Baby was born by Emergency LSCS because of premature rupture of membranes and absent liquor with birth weight of $2.19 \mathrm{~kg}$. Baby required intubation and surfactant at admission for RDS. Baby was extubated next day.On examination baby was noticed to have depressed nasal bridge, hypertelorism, high forehead,bilateral reducible inguinal hernias,posterior plagiocephaly with hypotonia.A normal male genitalia with both testis in scrotum identified. Hypotonia persisted with poor feeding requiring NG feeds.X rays showed thoracic scoliosis with curvature convex to right along with hemivertebrae T4-T9. Infant was also having left sided hydronephrosis with hydroureter and normal right kidney.He had normal newborn hearing screen.Ophthalmologic examination showed underdeveloped macula and hypoplasia of fovea.MRI examination showed evidence of Agenesis of corpus collasum.In view of multiple congenital anomalies microarray was requested that confirmed the diagnosis of $8 \mathrm{p} 23$ deletion. There was no famliy history of note.Currently baby is 14 weeks old and still requiring nasograstic feeds.

Discussion $8 \mathrm{p} 23$ deletion is a rare syndrome that was first described in 1976 by Weleber et.al.It has an estimated prevalence rate of about 1:20000 newborns.Patients display moderate to severe developmental delay,characteristic facial features, congenital heart defects,hypotonia,skeletal abnormalities such as scoliosis and agenesis of the corpus collasum. Prognosis is mainly dependant upon the severity of the signs and symptoms and its associated complications. 


\section{REFERENCES}

1. Weleber RG,Verma RS,Kimberling WJ,Lubs HA.Duplication-defeciency of the short arm of chromosome 8 following artificial insemination.Ann Genet1976;19 (4):241-247

2. Fryns JP.8p inverted duplication/deletion syndrome.(updated 2013Jan;cited 2013 May).

3. Knijenenburg J,van Haeringen $A$, Hansson $\mathrm{KB}$, et al.Ring chromosome formation as a novel escape mechanism in patientswith inverted duplication and terminal deletion.Eur J Hum Genet 2007;15(5):548-555.

\section{P141 A RETROSPECTIVE AUDIT OF CHRONIC URTICARIA IN THE IRISH PAEDIATRIC POPULATION}

${ }^{1}$ Kaie Rosborough* ${ }^{1}$ Connor Keller, ${ }^{1}$ Jillian Jung, ${ }^{1}$ Aisling O'Grady Walshe, ${ }^{1}$ Stuart Lockhart, ${ }^{1}$ Conor Hughes, ${ }^{2,3}$ Aideen Byrne. ${ }^{1}$ School of Medicine, Trinity College Dublin, Dublin, Ireland; ${ }^{2}$ Paediatric Allergy, Tallaght Hospital, Dublin, Ireland; ${ }^{3}$ Department of Allergy, Our Lady Children's Hospital Crumlin, Dublin, Ireland

10.1136/archdischild-2019-epa.496

Background Non-allergic Chronic Urticaria (CU), which includes both Chronic Spontaneous Urticaria (CSU) and Chronic Inducible Urticaria (CIU), is a condition characterised by wheals, erythema and pruritus. It is often associated with angioedema. CSU/CIU are often misinterpreted as an allergic condition by both families and healthcare professionals leading to unnecessary investigations, unwarranted therapeutics, and a high referral burden on paediatric allergy specialists. The aim of this study was to define the demographics of cases of chronic urticaria referred to an Irish allergy clinic and to explore diagnostic and treatment patterns at a primary care level. Finally, this study aimed to determine the specialist management strategy as compared to the current British Society for Allergy and Clinical Immunology (BSACI) guidelines.

Methods A retrospective audit of medical records of all children seen by the Allergy service at Our Lady's Children's Hospital Crumlin (OLCHC) with a diagnosis of Chronic Spontaneous Urticaria or Chronic Inducible Urticaria between January 2014 - December 2017 was conducted.

Results A total of 97 children were included. Both genders were equally affected (Males $n=48$ ) and the most common age of onset of CU was -5 years $(n=44)$. Of the 97 children reviewed, the majority (71\%) presented through primary care referral $(n=69)$. At the primary care level, only $10 \%$ of children $(n=10)$ were appropriately diagnosed with CU. The most common pre-referral misdiagnosis was allergy $(n=32)$, followed by no diagnosis $(n=12)$. While $65 \%$ of children $(n=63)$ were started on an antihistamine, only $32 \% \quad(n=28)$ received a second-generation $\mathrm{H}_{1}$ antihistamine by the primary care provider. Five cases inappropriately were prescribed oral or topical corticosteroids. Once reviewed by the specialist allergy service at OLCHC, $100 \%$ of the children were prescribed second-generation $\mathrm{H}_{1}$ antihistamines, primarily cetirizine $(n=64)$, and no children were treated with corticosteroids. Following the first allergy consult, $70 \%$ of children saw resolution of symptoms and were discharged $(\mathrm{n}=67)$.

Conclusion This audit demonstrates that recognition and appropriate treatment of Chronic Spontaneous and Chronic Inducible Urticaria are key targets for primary care education in order to achieve prompt resolution of symptoms and reduced referral burden on specialist services.

\section{P142 \\ EFFECTIVENESS AND RELIABILITY OF PRE-DISCHARGE JAUNDICE ASSESSMENT TOOLS: A QUALITY IMPROVEMENT PROJECT}

${ }^{1}$ Liqa ur Rehman*, ${ }^{2}$ Mushtaq Hussein, ${ }^{2}$ Naeem Shori, ${ }^{2}$ Riaz Ahmed, ${ }^{2}$ Muhammad Zia, ${ }^{2}$ Muhammad Azam. 'Portiuncula University Hospital, Ballinasloe, Ireland; ' ${ }^{1}$ Wexford General Hospital, Wexford, Ireland

\subsection{6/archdischild-2019-epa.497}

Background Visual inspection using the Kramer index was the only tool available to detect the degree of jaundice at our hospital.A retrospective audit done at this hospital showed that only $13 \%$ of neonates required phototherapy and $87 \%$ were pricked unnecessarily,adding to parental anxiety.Therefore,we decided to launch a one-year prospective study (July2016-2017).

Aim To determine the diagnostic accuracy of two pre-discharge jaundice assessment tools and to correlate these measurements with SBR results in neonates $>35$ weeks in order to implement a safe,cost-effective\&patiently friendly alternative to improve quality of care.

Method All health care professionals were briefed on the guidelines set out in $\mathrm{NICE}^{4} \& \mathrm{AAP}^{1}$ for visual assessment of jaundice.In the first 6 months, any neonate being visually assessed as significantly jaundiced (below nipple line) was referred for SBR intake.Results were then plotted on nomograms for further action. ${ }^{1,3,4}$

During the next six months, a TcB was purchased and all health care professionals were briefed on equipment usage and criteria for further action ${ }^{1,3,4}$.A pre-discharge TcB check was performed on all neonates. SBR was taken within an hour of TcB if it measured $>200 \mu \mathrm{mol} / 1$ in term $\&>170 \mu \mathrm{mol} / \mathrm{l}$ in late pre-term neonates ${ }^{1,3,4}$.Further follow up was arranged as per risk designation on nomogram (Recommendation 3.0, 6.12, AAP).

The correlation of the above variables was analysed.Reliability of $\mathrm{TcB}$ was then determined in both terms and pre-terms.

Inclusion criteria: Age: $<14$ days, $>38$ weeks $\&>24$ hoursofage, between 35-37+6weeks $\&>48$ hoursofage ${ }^{1,3,4}$

Exclusion criteria: $<35$ weeksgestation, risk factors as listed in $\mathrm{AAP}(\text { Figure } 3)^{1,3,4}$.

Results

\begin{tabular}{lll}
\multicolumn{2}{l}{ Abstract P142 } & Table 1 \\
& First six months & Second six months \\
& (Visual assessment) & (Transcutaneous Billinometer) \\
\hline Sample size & 842 & 820 \\
Referred for SBR & 123 & 41 \\
Phototherapy & 13 & 10 \\
Re-admission & 5 & 0 \\
\hline
\end{tabular}

Reliability of TcB:Sample size:72

Average difference: Term $\pm 22 \mu \mathrm{mol} / 1$, Pre-term $\pm 29 \mu \mathrm{mol} / \mathrm{l}$

Conclusion Visual estimation of bilirubin levels for the degree of jaundice can lead to errors with $70 \%$ sensitivity and $89 \%$ specificity.TcB,however,is a non-invasive, cost effective and reliable alternative tool with $100 \%$ sensitivity and $96 \%$ specificity. $\mathrm{TcB}>200 \mu \mathrm{mol} / 1$ in term and $>170 \mu \mathrm{mol} / \mathrm{l}$ in late pre-terms is 